\title{
Fuzzy parametrized fuzzy soft topology
}

\author{
Idris Zorlutuna and Serkan Atmaca \\ Department of Mathematics, Cumhuriyet University, Sivas, Turkey \\ Received: 19 October 2015, Revised: 21 October 2015, Accepted: 28 November 2015 \\ Published online: 30 January 2016.
}

\begin{abstract}
Recently, researches have contributed a lot towards fuzzification of Soft Set Theory. In this paper, we introduce the topological structure of fuzzyfying soft sets called fuzzy parametrized fuzzy soft sets. We define the notion of quasi-coincidence for fuzzy parametrized fuzzy soft sets and investigated basic properties of it. We study the closure, interior, base, continuity and compactness in the content of fuzzy parametrized fuzzy soft topological spaces.
\end{abstract}

Keywords: Fuzzy parametrized fuzzy soft set, fuzzy parametrized fuzzy soft mapping, topology

\section{Introduction}

In 1965, Zadeh [32] generalized the usual notion of a set with the introduction of fuzzy set. The theory of fuzzy set has been successfully applied to many areas such as many real life problems in uncertain, ambiguous environment. Chang [15] defined the fuzzy topology and introduced many topological notions in fuzzy setting, in 1968.

In 1999, Molodtsov [26] introduced the soft set theory which is a new approach for modelling uncertainty and presented that soft set can be applied to several areas, such as game theory, perron integrations, smoothness of functions and so on. Many researchers successfully improved the theory by applying this concept on topological spaces (e.g. [6,7, 19,27,33]), group theory, ring theory (e.g. [1,2,14,17,21]), and also decision making problems (e.g. [12, 13, 16, 23]).

Recently, researchers have combined fuzzy set and soft set to generalize the spaces and to solve more complicated problems. By this way, many interesting applications of soft set theory have been expanded. First combination of fuzzy set and soft set is fuzzy soft set and it was given by Maji and et al [24]. Then fuzzy soft set theory has been applied in several directions, such as topology (e.g. [3,5,29,30]), various algebraic structures (e.g. [4,20]) and especially decision making (e.g. [18,22,28,31]). Second combination of fuzzy set and soft set was given by Çă̆man and et al. [8] and called it as fuzzy parametrized soft set (as shortly FPS set). In that paper, Çağman and et al. defined operations on FPS sets and improved several results. After that, Çağman and Deli $[9,11]$ applied FPS sets to define some decision making methods and applied these methods to problems that contain uncertainties and fuzzy object. The third and the last one was also given by Çağman and et al. [10] and it is called fuzzy parametrized fuzzy soft set (as shortly FPFS set). Then they defined operations on FPFS sets and improved an method to solve some decision making problems.

In the present paper, we consider the topological structure of FPFS sets. Firstly, we give some basic ideas of FPFS sets and also studied results. We define FPFS quasi-coincidence, as a generalization of quasi-coincidence in fuzzy manner 
[25] and use this notion to characterize concepts of FPFS closure and FPFS base in FPFS topological spaces. We also introduce the notion of mapping on FPFS classes and investigate the properties of FPFS images and FPFS inverse images of FPFS sets. We define FPFS topology in Chang's sense. We study the FPFS closure and FPFS interior operators and properties of these concepts. Lastly we define FPFS continuous mappings and we show that image of a FPFS compact space is also FPFS compact.

This paper is the fundamental study on FPFS topological spaces. One can use results deducted from this paper in the theory topological structures.

\section{Preliminaries}

Throughout this paper $X$ denotes initial universe, $E$ denotes the set of all possible parameters which are attributes, characteristic or properties of the objects in $X$, and the set of all subsets of $X$ will be denoted by $P(X)$.

Definition 1. [32] A function A from $X$ to unit interval $[0,1]$ is called fuzzy set in $X$. For every $x \in A, \mu_{A}(x)$ is called the grade of membership of $x$ in A. A fuzzy point in $X$, whose value is $\alpha(0<\alpha \leq 1)$ at the support $x \in X$, is denoted by $x_{\alpha}$. $A$ fuzzy point $x_{\alpha} \in A$, where $A$ is fuzzy set in $X$ iff $\alpha \leq \mu_{A}(x)$. A is called empty fuzzy set if $\mu_{A}(x)=0$ for all $x \in X$, denoted by $\overline{0}$. If $\mu_{A}(x)=1$ for all $x \in X$, A is denoted by $\overline{1}$.

Definition 2. [26] A pair $(F, E)$ is called a soft set over $X$ if $F$ is a mapping defined by $F: E \rightarrow P(X)$.

In the other words, a soft set is a parametrized family of subsets of the set X. For each e $\in E$, the set $F(e)$ may be considered as the set of e-elements of the soft set $(F, E)$.

Definition 3. [10] Let A be a fuzzy set over E. A fuzzy parametrized fuzzy soft set (FPFS) $F_{A}$ on the universe X is defined as follows:

$$
F_{A}=\left\{\left(\mu_{A}(e) / e, f_{A}(e)\right): e \in E, f_{A}(e) \in I^{X}, \mu_{A}(e) \in[0,1]\right\}
$$

where the function $f_{A}: E \rightarrow I^{X}$ is called approximate function of $F_{A}$ such that $f_{A}(e)=\overline{0}$ if $\mu_{A}(e)=0$.

From now on, the set of all FPFS sets over $X$ will be denoted by $\operatorname{FPF}(X, E)$.

Definition 4. [10] Let $F_{A} \in F P F S(X, E)$.

(1) $F_{A}$ is called the empty FPFS set if $\mu_{A}(e)=0$ and $f_{A}(e)=\overline{0}$ for all every $e \in E$, denoted by $F_{\varnothing}$.

(2) $F_{A}$ is called A-universal FPFS set if $\mu_{A}(e)=1$ and $f_{A}(e)=\overline{1}$ for all $e \in A$, denoted by $F_{\widetilde{A}}$. If $A=E$, then A-universal FPFS set is called universal FPFS set, denoted by $F_{\widetilde{E}}$.

Definition 5. [10] Let $F_{A}, F_{B} \in F P F S(X, E)$.

(1) $F_{A}$ is called a subset of $F_{B}$ if $A \leq B$ and $f_{A}(e) \leq f_{B}(e)$ for every $e \in E$ and we write $F_{A} \widetilde{\subset} F_{B}$.

(2) $F_{A}$ and $F_{B}$ are said to be equal, denoted by $F_{A}=F_{B}$ if $F_{A} \widetilde{\subset} F_{B}$ and $F_{B} \widetilde{\subset} F_{A}$.

(3) The union of $F_{A}$ and $F_{B}$, denoted by $F_{A} \widetilde{\cup} F_{B}$, is the FPFS set, defined by the membership and approximate functions $\mu_{A \cup B}(e)=\max \left\{\mu_{A}(e), \mu_{B}(e)\right\}$ and $f_{A \cup B}(e)=f_{A}(e) \vee g_{B}(e)$ for every $e \in E$, respectively.

(4) The intersection of $F_{A}$ and $F_{B}$, denoted by $F_{A} \widetilde{\cap} G_{B}$, is the FPFS set, defined by the membership and approximate functions $\mu_{A \cap B}(e)=\min \left\{\mu_{A}(e), \mu_{B}(e)\right\}$ and $f_{A \cap B}(e)=f_{A}(e) \wedge g_{B}(e)$ for every $e \in E$, respectively. 
Definition 6. [10] Let $F_{A} \in F P F S(X, E)$. Then the complement of $F_{A}$, denoted by $F_{A}^{c}$, is the FPFS set, defined by the membership and approximate functions $\mu_{A^{c}}(e)=1-\mu_{A}(e)$ and $f_{A}^{c}(e)=\overline{1}-f_{A}(e)$ for every $e \in E$, respectively. Clearly,

$$
\left(F_{A}^{c}\right)^{c}=F_{A}, F_{\widetilde{E}}^{c}=F_{\varnothing} \text { and } F_{\varnothing}^{c}=F_{\widetilde{E}} .
$$

Proposition 1. [10] Let $F_{A}, F_{B}$ and $F_{C} \in F P F S(X, E)$. Then

(1) $\left(F_{A} \widetilde{\cup} F_{B}\right)^{c}=F_{A}^{c} \widetilde{\cap} F_{B}^{c}$.

(2) $\left(F_{A} \widetilde{\cap} F_{B}\right)^{c}=F_{A}^{c} \widetilde{\cup} F_{B}^{c}$.

(3) $F_{A} \widetilde{\cap} F_{A}=F_{A}, F_{A} \widetilde{\cup} F_{A}=F_{A}$.

(4) $F_{A} \widetilde{\cap} F_{\varnothing}=F_{\varnothing}, F_{A} \widetilde{\cap} F_{\widetilde{E}}=F_{A}$.

(5) $F_{A} \widetilde{\cap} F_{B}=F_{B} \widetilde{\cap} F_{A}, F_{A} \widetilde{\cup} F_{B}=F_{B} \widetilde{\cup} F_{A}$.

(6) $F_{A} \widetilde{\cap}\left(F_{B} \widetilde{\cap} F_{C}\right)=\left(F_{A} \widetilde{\cap} F_{B}\right) \widetilde{\cap} F_{C}, F_{A} \widetilde{\cup}\left(F_{B} \widetilde{\cup} F_{C}\right)=\left(F_{A} \widetilde{\cup} F_{B}\right) \widetilde{\cup} F_{C}$.

(7) $F_{A} \widetilde{\cup} F_{\varnothing}=F_{A}, F_{A} \widetilde{\cup} F_{\widetilde{E}}=F_{\widetilde{E}}$.

\section{Some properties of FPFS sets and FPFS mappings}

Definition 7. Let $J$ be an arbitrary index set and $F_{A_{i}} \in F P F S(X, E)$ for all $i \in J$.

(1) The union of $F_{A_{i}}$ 's, denoted by $\underset{i \in J}{\widetilde{J}} F_{A_{i}}$, is the FPFS set, defined by the membership and approximate functions $\mu_{i \in J} A_{i}(e)=\sup _{i \in J}\left\{\mu_{A_{i}}(e)\right\}$ and $f_{i \in J} A_{i}(e)=\vee_{i \in J} f_{A_{i}}(e)$ for every $e \in E$, respectively.

(2) The intersection of $F_{A_{i}}$ 's, denoted by $\underset{i \in J}{\widetilde{T}} F_{A_{i}}$, is the FPFS set, defined by the membership and approximate functions $\mu_{i \in J} A_{i}(e)=\inf _{i \in J}\left\{\mu_{A_{i}}(e)\right\}$ and $f_{i \in J} A_{i}(e)=\wedge_{i \in J} f_{A_{i}}(e)$ for every $e \in E$, respectively.

Proposition 2. Let $J$ be an arbitrary index set and $F_{A_{i}} \in F P F S(X, E)$ for all $i \in J$. Then

(1) $\left(\widetilde{\cup} F_{i \in J} F_{A_{i}}\right)^{c}=\underset{i \in J}{\widetilde{n}} F_{A_{i}}^{c}$.

(2) $\left(\widetilde{\cap}_{i \in J} F_{A_{i}}\right)^{c}=\underset{i \in J}{\widetilde{\cup}} F_{A_{i}}^{c}$.

Proof.

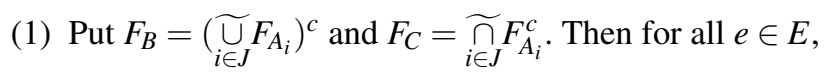

$$
\mu_{B}(e)=1-\mu_{i \in J} A_{i}(e)=1-\sup _{i \in J}\left\{\mu_{A_{i}}(e)\right\}=\inf _{i \in J}\left\{1-\mu_{A_{i}}(e)\right\}=\inf _{i \in J}\left\{\mu_{A_{i}^{c}}(e)\right\}=\mu_{C}(e)
$$

and

$$
f_{B}(e)=\overline{1}-f_{i \in J} A_{i}(e)=\overline{1}-\underset{i \in J}{\vee} f_{A_{i}}(e)=\underset{i \in J}{\wedge}\left(\overline{1}-f_{A_{i}}(e)\right)=\underset{i \in J}{\wedge} f_{A_{i}^{c}}(e)=f_{i \in J} A_{i}^{c}(e)=f_{C}(e) .
$$

This completes the proof. The other can be proved similarly

Definition 8. The FPFS set $F_{A} \in F P F S(X, E)$ is called FPFS point if $A$ is a fuzzy point in $E$ and $f_{A}(e)$ is a fuzzy point in $X$ for $e \in$ suppA. If $A=\{e\}, \mu_{A}(e)=\beta \in(0,1]$ and $f_{A}(e)(x)=\alpha \in(0,1]$, then we denote this FPFS point by $e_{x_{\alpha}}^{\beta}$.

Definition 9. Let $e_{x_{\alpha}}^{\beta}, F_{A} \in F P F S(X, E)$. We say that $e_{x_{\alpha}}^{\beta} \widetilde{\in} F_{A}$ read as $e_{x_{\alpha}}^{\beta}$ belongs to $F_{A}$ if $\beta \leq \mu_{A}(e)$ and $\alpha \leq f_{A}(e)(x)$.

Proposition 3. Every non empty FPFS set $F_{A}$ can be expresssed as the union of all the FPFS points which belong to $F_{A}$. 
Proof. This follows from the fact that any fuzzy set is the union of fuzzy points which belong to it [25].

Definition 10. Let $F_{A}, F_{B} \in F P F S(X, E) . F_{A}$ is said to be FPFS quasi-coincident with $F_{B}$, denoted by $F_{A} q F_{B}$, if there exists $e \in E$ such that $\mu_{A}(e)+\mu_{B}(e)>1$ or there exists $x \in X$ such that $f_{A}(e)(x)+f_{B}(e)(x)>1$. If $F_{A}$ is not FPFS quasi-coincident with $F_{B}$, then we write $F_{A} \bar{q} F_{B}$.

Definition 11. Let $e_{x_{\alpha}}^{\beta}, F_{A} \in F P F S(X, E) . e_{x_{\alpha}}^{\beta}$ is said to be FPFS quasi-coincident with $F_{A}$, denoted by $e_{x_{\alpha}}^{\beta} q F_{A}$, if $\beta+$ $\mu_{A}(e)>1$ or $\alpha+f_{A}(e)(x)>1$. If $e_{x_{\alpha}}^{\beta}$ is not FPFS quasi-coincident with $F_{A}$, then we write $e_{x_{\alpha}}^{\beta} \bar{q} F_{A}$.

Proposition 4. Let $F_{A}, F_{B} \in F P F S(X, E)$, Then the following are true.

(1) $F_{A} \widetilde{\simeq} F_{B} \Leftrightarrow F_{A} \bar{q} F_{B}^{c}$.

(2) $F_{A} q F_{B} \Rightarrow F_{A} \widetilde{\cap} F_{B} \neq F_{\varnothing}$.

(3) $F_{A} \bar{q} F_{A}^{c}$.

(4) $F_{A} q F_{B} \Leftrightarrow$ there exists an $e_{x_{\alpha}}^{\beta} \widetilde{\in} F_{A}$ such that $e_{x_{\alpha}}^{\beta} q F_{B}$.

(5) For all $e_{x_{\alpha}}^{\beta} \in \operatorname{FPFS}(X, E), e_{x_{\alpha}}^{\beta} \tilde{\in} F_{A}^{c} \Leftrightarrow e_{x_{\alpha}}^{\beta} \bar{q} F_{A}$.

(6) $F_{A} \widetilde{\widetilde{C}} F_{B} \Rightarrow$ If $e_{x_{\alpha}}^{\beta} q F_{A}$, then $e_{x_{\alpha}}^{\beta} q F_{B}$ for all $e_{x_{\alpha}}^{\beta} \in \operatorname{FPFS}(X, E)$.

Proof.

(1) $F_{A} \widetilde{\widetilde{\subseteq}} F_{B} \Leftrightarrow$ for all $e \in E, A \leq B$ and $f_{A}(e) \leq f_{B}(e)$

$\Leftrightarrow$ for all $e \in E$ and $x \in X, \mu_{A}(e) \leq \mu_{B}(e)$ and $f_{A}(e)(x) \leq f_{B}(e)(x)$

$\Leftrightarrow$ for all $e \in E$ and $x \in X, \mu_{A}(e)-\mu_{B}(e) \leq 0$ and $f_{A}(e)-f_{B}(e) \leq 0$

$\Leftrightarrow$ for all $e \in E$ and $x \in X, \mu_{A}(e)+1-\mu_{B}(e) \leq 1$ and $f_{A}(e)(x)+1-f_{B}(e)(x) \leq 1$

$\Leftrightarrow F_{A} \bar{q} F_{B}^{c}$.

(2) Let $F_{A} q F_{B}$. Then there exists an $e \in E$ and $x \in X$ such that $\mu_{A}(e)+\mu_{B}(e)>1$ or $f_{A}(e)(x)+f_{B}(e)(x)>1$. If $\mu_{A}(e)+$ $\mu_{B}(e)>1$, then $A \wedge B \neq \overline{0}$ and the proof is easy. If $f_{A}(e)(x)+f_{B}(e)(x)>1$, then $f_{A}(e) \wedge f_{B}(e) \neq \overline{0}$. Hence $F_{A} \widetilde{\cap} F_{B} \neq F_{\varnothing}$.

(3) Suppose that $F_{A} q F_{A}^{c}$. Then there exists $e \in E$ and $x \in X$ such that $\mu_{A}(e)+1-\mu_{A}(e)>1$ or $f_{A}(e)(x)+1-f_{A}(e)(x)>1$. This is contradiction.

(4) If $F_{A} q F_{B}$, then there exist an $e \in E$ and $x \in X$ such that $\mu_{A}(e)+\mu_{B}(e)>1$ or $f_{A}(e)(x)+f_{B}(e)(x)>1$ Put $\beta=\mu_{A}(e)$ and $\alpha=f_{A}(e)(x)$. Then we have $e_{x_{\alpha}}^{\beta} \tilde{\in} F_{A}$ and $e_{x_{\alpha}}^{\beta} q F_{B}$.

Conversely, let $e_{x_{\alpha}}^{\beta} \widetilde{\in} F_{A}$ with $e_{x_{\alpha}}^{\beta} q F_{B}$. Then $\beta \leq \mu_{A}(e), \alpha \leq f_{A}(e)(x)$. Since $e_{x_{\alpha}}^{\beta} q F_{B}, \beta+\mu_{B}(e)>1$ or $\alpha+f_{B}(e)(x)>1$. Therefore, we have $\mu_{A}(e)+\mu_{B}(e)>1$ or $f_{A}(e)(x)+f_{B}(e)(x)>1$. This shows that $F_{A} q F_{B}$.

(5) It is obvious from (1).

(6) Let $e_{x_{\alpha}}^{\beta}, F_{A} \in F P F S(X, E)$ and $e_{x_{\alpha}}^{\beta} q F_{A}$. Then $\beta+\mu_{A}(e)>1$ or $\alpha+f_{A}(e)(x)>1$. Since $F_{A} \widetilde{\widetilde{C}} F_{B}, \beta+\mu_{B}(e)>1$ or $\alpha+f_{B}(e)(x)>1$. Hence we have $e_{x_{\alpha}}^{\beta} q F_{B}$.

Proposition 5. Let $\left\{F_{A_{i}}: i \in J\right\}$ be a family of FPFS sets in FPFS $(X, E)$ where $J$ is an index set. Then $e_{x_{\alpha}}^{\beta}$ is quasicoincident with $\widetilde{\cup}_{i \in J} F_{A_{i}}$ if and only if there exists some $F_{A_{i}} \in\left\{F_{A_{i}}: i \in J\right\}$ such that $e_{x_{\alpha}}^{\beta} q F_{A_{i}}$.

Proof. Obvious.

Definition 12. Let FPFS $(X, E)$ and $F P F S(Y, K)$ be families of all FPFS sets over $X$ and $Y$, respectively. Let $u: X \rightarrow Y$ and $p: E \rightarrow K$ be two functions. Then a FPFS mapping $f_{u p}: F P F S(X, E) \rightarrow F P F S(Y, K)$ is defined as: 
(1) for $F_{A} \in F P F S(X, E)$, the image of $F_{A}$ under the $f_{u p}$ is the FPFS set $G_{S}$ over $Y$ defined by the approximate function, $\forall k \in K$ and,

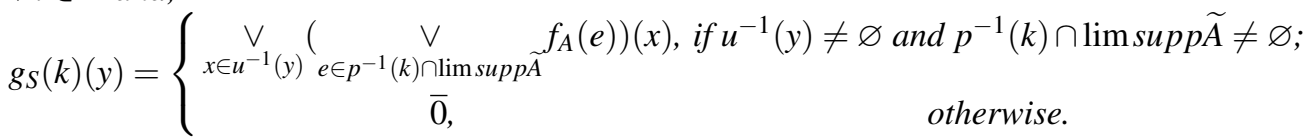

where $p(A)=S$ is fuzzy set in $K$.

(2) for $G_{S} \in F P F S(Y, K)$, then the pre-image of $G_{S}$ under the $f_{u p}$ is the FPFS set $F_{A}$ over $X$ defined by the approximate function, $\forall e \in E . f_{A}(e)(x)=g_{S}(p(e))(u(x))$ where $A=p^{-1}(S)$ is fuzzy set in $E$.

If $u$ and $p$ is injective, then the FPFS mapping $f_{u p}$ is said to be injective. If $u$ and $p$ is surjective, then the FPFS mapping $f_{u p}$ is said to be surjective. The FPFS mapping $f_{u p}$ is called constant, if $u$ and $p$ are constant.

Theorem 1. Let $X$ and $Y$ crips sets $F_{A}, F_{A_{i}} \in F P F S(X, E), G_{S}, G_{S_{i}} \in F P F S(Y, K) \forall i \in J$, where $J$ is an index set. Let $f_{u p}: F P F S(X, E) \rightarrow F P F S(Y, K)$ be a FPFS mapping. Then,

(1) If $F_{A_{1}} \widetilde{\subset} F_{A_{2}}$ then $f_{u p}\left(F_{A_{1}}\right) \widetilde{\subset} f_{u p}\left(F_{A_{2}}\right)$.

(2) If $G_{S_{1}} \widetilde{\subset} G_{S_{2}}$ then $f_{u p}^{-1}\left(G_{S_{1}}\right) \widetilde{\subset} f_{u p}^{-1}\left(G_{S_{2}}\right)$.

(3) $F_{A} \widetilde{\subset} f_{u p}^{-1}\left(f_{u p}\left(F_{A}\right)\right)$, the equality holds if $f_{u p}$ is injective.

(4) $f_{u p}\left(f_{u p}^{-1}\left(G_{S}\right)\right) \widetilde{\subset} G_{S}$, the equality holds if $f_{u p}$ is surjective.

(5) $f_{u p}\left(\widetilde{\cup}_{i \in J} F_{A_{i}}\right)=\widetilde{\cup}_{i \in J} f_{u p}\left(F_{A_{i}}\right)$.

(6) $f_{u p}\left(\widetilde{\cap}_{i \in J} F_{A_{i}}\right) \widetilde{\complement} \widetilde{\cap}_{i \in J} f_{u p}\left(F_{A_{i}}\right)$, the equality holds if $f_{u p}$ is injective.

(7) $f_{u p}^{-1}\left(\widetilde{\cup}_{i \in J} G_{S_{i}}\right)=\widetilde{\cup}_{i \in J} f_{u p}^{-1}\left(G_{S_{i}}\right)$.

(8) $f_{u p}^{-1}\left(\widetilde{\cap}_{i \in J} G_{S_{i}}\right)=\widetilde{\cap}_{i \in J} f_{u p}^{-1}\left(G_{S_{i}}\right)$.

(9) $\left(f_{u p}^{-1}\left(G_{S}\right)\right)^{c}=f_{u p}^{-1}\left(G_{S}^{c}\right)$.

(10) $f_{u p}^{-1}\left(G_{\widetilde{K}}\right)=F_{\widetilde{E}}$.

(11) $f_{u p}^{-1}\left(G_{\varnothing}\right)=F_{\varnothing}$.

(12) $f_{\text {up }}\left(F_{\widetilde{E}}\right) \widetilde{\subset} G_{\widetilde{K}}$, the equality holds if $f_{u p}$ is surjective.

(13) $f_{u p}\left(F_{\varnothing}\right)=G_{\varnothing}$.

Proof. We only prove (3),(5),(7),(9),(10) and (11). The others can be proved similarly.

(3) Put $G_{S}=f_{u p}\left(F_{A}\right)$ and $F_{B}=f_{u p}^{-1}\left(G_{S}\right)$. Since $A \leq p^{-1}(p(A))=p^{-1}(S)=B$, it is sufficient to show $f_{A}(e) \leq f_{B}(e)$ for all $e \in E$ and $x \in X$

$$
\begin{aligned}
& f_{B}(e)(x)=g_{S}(p(e))(u(x)) \\
& =\underset{x \in u^{-1}(u(x))}{\vee}\left(\underset{e \in p^{-1}(p(e)) \cap \lim \operatorname{supp} \widetilde{A}}{\vee} f_{A}(e)\right)(x) \\
& \geq f_{A}(e)(x)
\end{aligned}
$$

This completes the proof.

(5) Put $G_{S_{i}}=f_{u p}\left(F_{A_{i}}\right)$ and $G_{S}=f_{u p}\left(\widetilde{\cup}_{i \in J}\left(F_{A_{i}}\right)\right)$. Then $S=p\left(\vee A_{i}\right)=\vee p\left(A_{i}\right)=\vee S_{i}$ and for all $k \in K$ and $y \in Y$, 


$$
\begin{aligned}
& g_{B}(k)(y)= \begin{cases}\underset{x \in u^{-1}(y)}{\vee}\left(\underset{e \in p^{-1}(k) \cap \lim \operatorname{supp} \tilde{A}}{\vee}\left(\bigvee_{i \in J}^{\vee} f_{A_{i}}(e)\right)(x)\right) & ; \text { if } u^{-1}(y) \neq \varnothing, p^{-1}(k) \cap \operatorname{limsupp} \widetilde{A} \neq \varnothing \\
0 & ; \text { otherwise }\end{cases} \\
& = \begin{cases}\underset{x \in u^{-1}(y)}{\vee}\left(\underset{e \in p^{-1}(k) \cap \lim \text { supp } \widetilde{A}}{\vee}\left(\underset{i \in J}{\vee}\left(f_{A_{i}}(e)\right)(x)\right)\right) & ; \text { if } u^{-1}(y) \neq \varnothing, p^{-1}(k) \cap \lim \operatorname{supp} \widetilde{A} \neq \varnothing \\
0 & ; \text { otherwise }\end{cases} \\
& = \begin{cases}\underset{i \in J}{\vee}\left(\underset{x \in u^{-1}(y)}{\vee}\left(\underset{e \in p^{-1}(k) \cap \lim \text { supp } \widetilde{A}}{\vee}\left(f_{A_{i}}(e)\right)(x)\right)\right. & ; \text { if } u^{-1}(y) \neq \varnothing, p^{-1}(k) \cap \lim \operatorname{supp} \widetilde{A} \neq \varnothing \\
0 & ; \text { otherwise }\end{cases} \\
& =\underset{i \in J}{\vee}\left\{\begin{array}{l}
\underset{x \in u^{-1}(y)}{\vee}\left(\underset{e \in p^{-1}(k) \cap \lim \operatorname{supp} \widetilde{A}}{\vee}\left(f_{A_{i}}(e)\right)(x)\right. \\
0 \quad \text { if } u^{-1}(y) \neq \varnothing, p^{-1}(k) \cap \lim \operatorname{supp} \widetilde{A} \neq \varnothing \\
\text {; otherwise }
\end{array}\right. \\
& =\left(\vee_{i \in J} g_{B_{i}}(k)\right)(y)
\end{aligned}
$$

This completes the proof.

(7) Put $F_{A_{i}}=f_{u p}^{-1}\left(G_{S_{i}}\right)$ and $F_{A}=f_{u p}^{-1}\left(\widetilde{\cup}_{i \in J} G_{S_{i}}\right)$. Then $A=p^{-1}\left(\vee S_{i}\right)=\vee p^{-1}\left(S_{i}\right)=\vee A_{i}$ and for all $e \in E$ and $x \in X$,

$$
\begin{aligned}
f_{A}(e)(x) & =\underset{i \in J}{\vee} g_{S_{i}}(p(e))(u(x)) \\
& =\bigvee_{i \in J}\left(g_{S_{i}}(p(e))(u(x))\right) \\
& =\underset{i \in J}{\vee} f_{A_{i}}(e)(x)
\end{aligned}
$$

This completes the proof.

(9) Put $f_{u p}^{-1}\left(G_{S}\right)=F_{A}$ and $f_{u p}^{-1}\left(G_{S}^{c}\right)=F_{B}$. Then for all $e \in E$ and $x \in X$,

$$
f_{B}(e)(x)=f_{p^{-1}\left(S^{c}\right)}(e)(x)=f_{\left(p^{-1}(S)\right)^{c}}(e)(x)=f_{A^{c}}(e)(x)
$$

where $p^{-1}(S)$ and $p^{-1}\left(S^{c}\right)$ are fuzzy sets in $E$. This shows that the approximate functions of $F_{B}$ and $F_{A}^{c}$ are equal. This completes the proof.

(10) Put $F_{A}=f_{u p}^{-1}\left(G_{\widetilde{K}}\right)$. Then for all $e \in E$ and $\left.x \in X, f_{A}(e)(x)=g_{\widetilde{K}}(p(e))(u(x))\right)=1$. This shows that $F_{A}=F_{\widetilde{E}}$.

(11) Since $p^{-1}(K)$ is fuzzy empty set i.e. $\overline{0}$, the proof is clear.

\section{FPFS topological spaces}

Definition 13. A FPFS topological space is a pair $(X, \tau)$ where $X$ is a nonempty set and $\tau$ is a family of FPFS sets over $X$ satisfying the following properties:

(T1) $F_{\varnothing}, F_{\widetilde{E}} \in \tau$.

(T2) If $F_{A}, F_{B} \in \tau$, then $F_{A} \widetilde{\cap} F_{B} \in \tau$.

(T3) If $F_{A_{i}} \in \tau, \forall i \in J$, then $\widetilde{\cup}_{i \in J} F_{A_{i}} \in \tau$.

Then $\tau$ is called a FPFS topology on X. Every member of $\tau$ is called FPFS open in $(X, \tau) . F_{B}$ is called FPFS closed in $(X, \tau)$ if $F_{B}^{c} \in \tau$. 
Example 1. The families $\tau_{\text {indiscrete }}=\left\{F_{\varnothing}, F_{\widetilde{E}}\right\}$ and $\tau_{\text {discrete }}=F P F S(X, E)$ are FPFS topology on $X$.

Example 2. Assume that $X=\left\{x_{1}, x_{2}, x_{3}, x_{4}\right\}$ is a universal set and $E=\left\{e_{1}, e_{2}, e_{3}\right\}$ is a set of parameters. If

$$
\begin{aligned}
& F_{A_{1}}=\left\{\left(\left(e_{1}\right)_{0,2},\left\{\left(x_{1}\right)_{0,3},\left(x_{3}\right)_{0,5}\right\}\right),\left(\left(e_{2}\right)_{0,3},\left\{\left(x_{1}\right)_{0,7},\left(x_{4}\right)_{0,6}\right\}\right),\left(\left(e_{3}\right)_{0,4},\left\{\left(x_{2}\right)_{0,2}\right\}\right)\right\}, \\
& F_{A_{2}}=\left\{\left(\left(e_{1}\right)_{0,2},\left\{\left(x_{1}\right)_{0,3},\left(x_{2}\right)_{0,7},\left(x_{3}\right)_{0,6}\right\}\right),\left(\left(e_{2}\right)_{0,5},\left\{\left(x_{1}\right)_{0,7},\left(x_{4}\right)_{0,6}\right\}\right),\left(\left(e_{3}\right)_{0,4},\left\{\left(x_{1}\right)_{0,8},\left(x_{2}\right)_{0,5}\right\}\right)\right\}, \\
& F_{A_{3}}=\left\{\left(\left(e_{1}\right)_{0,7},\left\{\left(x_{1}\right)_{1},\left(x_{3}\right)_{0,5}\right\}\right),\left(\left(e_{2}\right)_{0,3}, 1_{X}\right)_{1},\left(\left(e_{3}\right)_{0,9},\left\{\left(x_{2}\right)_{0,2},\left(x_{3}\right)_{0,9}\right\}\right)\right\}, \\
& F_{A_{4}}=\left\{\left(\left(e_{1}\right)_{0,7},\left\{\left(x_{1}\right)_{1},\left(x_{2}\right)_{0,7},\left(x_{3}\right)_{0,6}\right\}\right),\left(\left(e_{2}\right)_{0,5}, 1_{X}\right),\left(\left(e_{3}\right)_{0,9},\left\{\left(x_{1}\right)_{0,8},\left(x_{2}\right)_{0,5},\left(x_{3}\right)_{0,9}\right\}\right)\right\},
\end{aligned}
$$

then $\tau=\left\{F_{\varnothing}, F_{A_{1}}, F_{A_{2}}, F_{A_{3}}, F_{A_{4}}, F_{\widetilde{E}}\right\}$ is a FPFS topology on $X$.

Theorem 2. Let $(X, \tau)$ be a FPFS topological space and $\tau^{\prime}$ be family of all FPFS closed sets. Then;

(1) $F_{\varnothing}, F_{\widetilde{E}} \in \tau^{\prime}$

(2) If $F_{A}, F_{B} \in \tau^{\prime}$, then $F_{A} \widetilde{\cup} F_{B} \in \tau^{\prime}$,

(3) If $F_{A_{i}} \in \tau^{\prime}, \forall i \in J$, then $\widetilde{\cap}_{i \in J} F_{A_{i}} \in \tau^{\prime}$.

Proof. Straightforward.

Definition 14. Let $(X, \tau)$ be a FPFS topological space and $F_{A} \in F P F S(X, E)$. The FPFS closure of $F_{A}$ in $(X, \tau)$, denoted by $\overline{F_{A}}$, is the intersection of all FPFS closed supersets of $F_{A}$.

Clearly, $\overline{F_{A}}$ is the smallest FPFS closed set over $X$ which contains $F_{A}$.

Theorem 3. Let $(X, \tau)$ be a FPFS topological space and $F_{A}, F_{B} \in F P F S(X, E)$. Then,

(1) $\overline{F_{\varnothing}}=F_{\varnothing}$ and $\overline{F_{\widetilde{E}}}=F_{\widetilde{E}}$.

(2) $F_{A} \widetilde{\subset} \overline{F_{A}}$.

(3) $\overline{\overline{F_{A}}}=\overline{F_{A}}$.

(4) If $F_{A} \widetilde{\subset} F_{B}$, then $\overline{F_{A}} \widetilde{\subset} \overline{F_{B}}$.

(5) $F_{A}$ is a FPFS closed set if and only if $F_{A}=\overline{F_{A}}$.

(6) $\overline{F_{A} \widetilde{\cup} F_{B}}=\overline{F_{A}} \widetilde{\cup} \overline{F_{B}}$.

Proof. The statements (1),(2),(3) and (4) are obvious from the definition of FPFS closure.

(5) Let $F_{A}$ be a FPFS closed set. Since $\overline{F_{A}}$ is the smallest FPFS closed set which contains $F_{A}$, then $\overline{F_{A}} \widetilde{\subset} F_{A}$. Therefore, we have $F_{A}=\overline{F_{A}}$.

(6) Since $F_{A} \widetilde{\subset} F_{A} \widetilde{\cup} F_{B}$ and $F_{B} \widetilde{\subset} F_{A} \widetilde{\cup} F_{B}$ by (4), $\overline{F_{A}} \widetilde{\subset} \overline{F_{A} \widetilde{\cup} F_{B}}, \overline{F_{B}} \widetilde{\subset} \overline{F_{A} \widetilde{\cup} F_{B}}$ and hence $\overline{F_{A}} \widetilde{\cup} \overline{F_{B}} \widetilde{\subset} \overline{F_{A} \widetilde{\cup} F_{B}}$.

Conversely, since $\overline{F_{A}}, \overline{F_{B}}$ are FPFS closed sets, $\overline{F_{A}} \widetilde{\cup} \overline{F_{B}}$ is a FPFS closed set. Again since $F_{A} \widetilde{\cup} F_{B} \widetilde{\subset} \overline{F_{A}} \widetilde{\cup} \overline{F_{B}}$ by (4), $\overline{F_{A}} \widetilde{\cup} F_{B} \widetilde{\subset} \overline{F_{A}} \widetilde{\cup} \overline{F_{B}}$.

Definition 15. Let $(X, \tau)$ be a FPFS topological space. A FPFS set $F_{A}$ in FPFS $(X, E)$ is called FPFS-Q-neighborhood (briefly, FPFS-Q-nbd) of a FPFS set $F_{B}$ if there exists a FPFS open set $F_{C}$ in $\tau$ such that $F_{B} q F_{C}$ and $F_{C} \widetilde{\subseteq} F_{A}$. 
Theorem 4. Let $e_{x_{\alpha}}^{\beta}, F_{A} \in F P F S(X, E)$. Then $e_{x_{\alpha}}^{\beta} \widetilde{\in} \overline{F_{A}}$ if and only if each FPFS-Q-nbd of $e_{x_{\alpha}}^{\beta}$ is FPFS quasi-coincident with $F_{A}$.

Proof. Let $e_{x_{\alpha}}^{\beta} \widetilde{\in} \overline{F_{A}}$. Suppose that $F_{C}$ is a FPFS-Q-nbd of $e_{x_{\alpha}}^{\beta}$ and $F_{C} \bar{q} F_{A}$. Then there exists a FPFS open set $F_{B}$ such that $e_{x_{\alpha}}^{\beta} q F_{B} \widetilde{\subseteq} F_{C}$. Since $F_{C} \bar{q} F_{A}$, by Proposition $4(1), F_{A} \widetilde{\subseteq} F_{C}^{c} \widetilde{\subseteq} F_{B}^{c}$. Again since $e_{x_{\alpha}}^{\beta} q F_{B}, e_{x_{\alpha}}^{\beta}$ does not belong to $F_{B}^{c}$. This is a contradiction with $\overline{F_{A}} \widetilde{\simeq} F_{B}^{c}$.

Conversely, let each Q-nbd of $e_{x_{\alpha}}^{\beta}$ be FPFS quasi-coincident with $F_{A}$. Suppose that $e_{x_{\alpha}}^{\beta}$ does not belong to $\overline{F_{A}}$. Then there exists a FPFS closed set $F_{B}$ which is containing $F_{A}$ such that $e_{x_{\alpha}}^{\beta}$ does not belong to $F_{B}$. By Proposition 4(5), we have $e_{x_{\alpha}}^{\beta} q F_{B}^{c}$. Then $F_{B}^{c}$ is a FPFS-Q-nbd of $e_{x_{\alpha}}^{\beta}$ and by Proposition $4(1), F_{A} \bar{q} F_{B}^{c}$. This is a contradiction with the hypothesis.

Definition 16. Let $(X, \tau)$ be a FPFS topological space and $F_{A} \in F P F S(X, E)$. The FPFS interior of $F_{A}$, denoted by $F_{A}^{\circ}$, is the union of all FPFS open subsets of $F_{A}$. Clearly, $F_{A}^{\circ}$ is the largest FPSFS open set contained in $F_{A}$.

Theorem 5. Let $(X, \tau)$ be a FPFS topological space and $F_{A}, F_{B} \in F P F S(X, E)$. Then,

(1) $\left(F_{\varnothing}\right)^{\circ}=F_{\varnothing}$ and $\left(F_{\widetilde{E}}\right)^{\circ}=F_{\widetilde{E}}$.

(2) $F_{A}^{\circ} \widetilde{\subset} F_{A}$.

(3) $\left(F_{A}^{\circ}\right)^{\circ}=F_{A}^{\circ}$.

(4) If $F_{A} \widetilde{\subset} F_{B}$, then $F_{A}^{\circ} \widetilde{\subset} F_{B}^{\circ}$.

(5) $F_{A}$ is a FPFS open set if and only if $F_{A}=F_{A}^{\circ}$.

(6) $\left(F_{A} \widetilde{\cap} F_{B}\right)^{\circ}=F_{A}^{\circ} \widetilde{\cap} F_{B}^{\circ}$.

Proof. Similar to that of Theorem 3.

Theorem 6. Let $(X, \tau)$ be a FPFS topological space and $F_{A} \in F P F S(X, E)$. Then,

(1) $\left(F_{A}^{\circ}\right)^{c}=\overline{F_{A}^{c}}$.

(2) $\left(\overline{F_{A}}\right)^{c}=\left(F_{A}^{c}\right)^{\circ}$.

Proof. We only prove (1). The other is similar.

$$
\begin{aligned}
\left(F_{A}^{\circ}\right)^{c} & =\left(\widetilde{\cup}\left\{F_{B} \mid F_{B} \in \tau, F_{A} \widetilde{\subset} F_{B}\right\}\right)^{c} \\
& =\widetilde{\cap}\left\{F_{B}^{c} \mid F_{B} \in \tau, F_{A} \widetilde{\subset} F_{B}\right\} \\
& =\widetilde{\cap}\left\{F_{B}^{c} \mid F_{B}^{c} \in \tau^{\prime}, F_{B}^{c} \widetilde{\subset} F_{A}^{c}\right\} \\
& =\overline{F_{A}^{c}}
\end{aligned}
$$

Definition 17. Let $(X, \tau)$ be a FPFS topological space. A subcollection $\mathscr{B}$ of $\tau$ is called a base for $\tau$ if every member of $\tau$ can be expressed as a union of members of $\mathscr{B}$.

Example 3. If we consider the FPFS topology $\tau$ in Example 2, then one easily see that the family $\mathscr{B}=\left\{F_{\varnothing}, F_{A_{1}}, F_{A_{2}}, F_{A_{3}}, F_{\widetilde{E}}\right\}$ is a basis for $\tau$.

Proposition 6. Let $(X, \tau)$ be a FPFS topological space and $\mathscr{B}$ is subfamily of $\tau$. $\mathscr{B}$ is a base for $\tau$ if and only if for each $e_{x_{\alpha}}^{\beta}$ in FPFS $(X, E)$ and for each FPFS open $Q$-nbd $F_{A}$ of $e_{x_{\alpha}}^{\beta}$, there exists a $F_{B} \in \mathscr{B}$ such that $e_{x_{\alpha}}^{\beta} q F_{B} \widetilde{\widetilde{\subseteq}} F_{A}$.

Proof. Let $\mathscr{B}$ be a base for $\tau, e_{x_{\alpha}}^{\beta} \tilde{\in} F \operatorname{PFS}(X, E)$ and $F_{A}$ be a FPFS open Q-nbd of $e_{x_{\alpha}}^{\beta}$. Then there exists a subfamily $\mathscr{B}^{\prime}$ of $\mathscr{B}$ such that $F_{A}=\widetilde{\cup}\left\{F_{B} \mid F_{B} \in \mathscr{B}^{\prime}\right\}$. Suppose that $e_{x_{\alpha}}^{\beta} \bar{q} F_{B}$ for all $F_{B} \in \mathscr{B}^{\prime}$. Then $\beta+\mu_{B}(e) \leq 1$ and $\alpha+f_{B}(e)(x) \leq 1$ 
for every $F_{B} \in \mathscr{B}^{\prime}$. But this is contradiction with $\mu_{A}(e)=\sup \left\{\mu_{B}(e) \mid F_{B} \in \mathscr{B}^{\prime}\right\}$ and $f_{A}(e)(x)=\sup \left\{f_{B}(e)(x) \mid F_{B} \in \mathscr{B}^{\prime}\right\}$.

Conversely, If $\mathscr{B}$ is not a base for $\tau$, then there exists a $F_{A} \in \tau$ such that $F_{C}=\widetilde{\cup}\left\{F_{B} \in \mathscr{B}: F_{B} \widetilde{\subseteq} F_{A}\right\} \neq F_{A}$. Since $F_{C} \neq F_{A}$, there exists $e \in E$ and $x \in X$ such that $\mu_{C}(e)<\mu_{A}(e)$ or $f_{C}(e)(x)<f_{A}(e)(x)$. Put $\beta=1-\mu_{C}(e)$ or $\alpha=1-f_{C}(e)(x)$. Then in both case, we obtain that $e_{x_{\alpha}}^{\beta} q F_{A}$ and $e_{x_{\alpha}}^{\beta} \bar{q} F_{C}$. Therefore, we have $e_{x_{\alpha}}^{\beta} \bar{q} F_{B}$ for all $F_{B} \in \mathscr{B}$ which contained in $F_{A}$. This is a contradiction.

Definition 18. Let $\left(X, \tau_{1}\right)$ and $\left(Y, \tau_{2}\right)$ be two FPFS topological spaces. A FPFS mapping $f_{u p}:\left(X, \tau_{1}\right) \rightarrow\left(Y, \tau_{2}\right)$ is called FPFS continuous if $f_{u p}^{-1}\left(G_{S}\right) \in \tau_{1}$, for all $G_{S} \in \tau_{2}$.

Example 4. Let $X=\left\{x_{1}, x_{2}, x_{3}\right\}, Y=\left\{y_{1}, y_{2}, y_{3}\right\}, E=\left\{e_{1}, e_{2}\right\}, K=\left\{k_{1}, k_{2}\right\}$ and $\tau_{1}=\left\{F_{\varnothing}, F_{\widetilde{E}}, F_{A}\right\}, \tau_{2}=\left\{\widetilde{0}_{K}, \widetilde{1}_{K}, G_{S}\right\}$ be FPFS topologies on $X$ and $Y$ respectively, where $F_{A}=\left\{\left(\left(e_{1}\right)_{0,3},\left\{\left(x_{2}\right)_{0,3},\left(x_{3}\right)_{0,5}\right\}\right),\left(\left(e_{2}\right)_{0,2},\left\{\left(x_{1}\right)_{0,7},\left(x_{2}\right)_{0,4}\right\}\right)\right\}, G_{S}=$ $\left\{\left(\left(k_{1}\right)_{0,2},\left\{\left(y_{1}\right)_{0,4},\left(y_{2}\right)_{0,7}\right\}\right),\left(\left(k_{2}\right)_{0,3},\left\{\left(y_{1}\right)_{0,3},\left(y_{3}\right)_{0,5}\right\}\right)\right\}$. Define $u: X \rightarrow Y$ and $p: E \rightarrow K$ as $u\left(x_{1}\right)=y_{2}, u\left(x_{2}\right)=y_{1}$, $u\left(x_{3}\right)=y_{3}$ and $p\left(e_{1}\right)=k_{2}, p\left(e_{2}\right)=k_{1}$. Then the FPFS mapping $f_{u p}:\left(X, \tau_{1}\right) \rightarrow\left(Y, \tau_{2}\right)$ is FPFS continuous.

Note that the constant mapping $f_{u p}:\left(X, \tau_{1}\right) \rightarrow\left(Y, \tau_{2}\right)$ is not continuous in general. As the following example shows.

Example 5. Let $X=\left\{x_{1}, x_{2}, x_{3}\right\}, Y=\left\{y_{1}, y_{2}\right\}, E=\left\{e_{1}, e_{2}\right\}, K=\left\{k_{1}, k_{2}\right\}$ and $\tau_{1}=\left\{F_{\varnothing}, F_{\widetilde{E}}\right\}, \tau_{2}=\left\{F_{\varnothing}, F_{\widetilde{K}}, G_{S}\right\}$ be topologies on $X$ and $Y$ respectively, where $G_{S}=\left\{\left(\left(k_{1}\right)_{0,2},\left\{\left(y_{1}\right)_{0,4},\left(y_{2}\right)_{1}\right\}\right),\left(\left(k_{2}\right)_{0,5},\left\{\left(y_{2}\right)_{0,7},\left(y_{3}\right)_{0,4}\right\}\right)\right\}$. Define $u: X \rightarrow Y$ and $p: E \rightarrow K$ as $u\left(x_{1}\right)=u\left(x_{2}\right)=u\left(x_{3}\right)=y_{2}$ and $p\left(e_{1}\right)=p\left(e_{2}\right)=k_{1}$. Then the FPFS mapping $f_{u p}:\left(X, \tau_{1}\right) \rightarrow\left(Y, \tau_{2}\right)$ is a constant FPFS mapping and is not continuous.

Let $\alpha \in[0,1]$. A constant fuzzy set on $E$ taking value $\alpha$ will be denoted by $\alpha_{E}$.

Definition 19. Let $F_{A} \in F P F S(X, E) . F_{A}$ is called $\alpha \beta-A$-universal FPFS set if $\mu_{A}(e)=\alpha$ and $f_{A}(e)=\beta_{X}$ for all $e \in A$, denoted by $F_{\widetilde{\alpha \beta}}$.

Definition 20. (see [5]) A FPFS topology is called enriched if it satisfies $F_{\widetilde{\alpha \beta}_{A}} \in \tau$ for all $\alpha \in(0,1]$ and $\beta \in(0,1]$.

Theorem 7. Let $\left(X, \tau_{1}\right)$ be a enriched FPFS topological space, $\left(Y, \tau_{2}\right)$ be a FPFS topological space and $f_{u p}: F P F S(X, E) \rightarrow F P F S(Y, K)$ be a constant FPFS mapping. Then $f_{u p}$ is FPFS continuous.

Proof. Let $u: X \rightarrow Y, p: E \rightarrow K$ be constant mapping defined as $u(x)=y_{0}, p(e)=k_{o}$ and $G_{S} \in \tau_{2}$. Put $f_{u p}^{-1}\left(G_{S}\right)=F_{A}$. Then $A=p^{-1}(S)=\alpha_{E}$ where $\alpha=\mu_{S}(k)$ and $f_{A}(e)(x)=g_{S}(p(e))(u(x))=g_{S}\left(k_{0}\right)\left(y_{0}\right)=\beta$ for all $e \in E$. Hence $F_{A}=F_{\widetilde{\alpha \beta}} \in \tau_{1}$ and so $f_{u p}:\left(X, \tau_{1}\right) \rightarrow\left(Y, \tau_{2}\right)$ is FPFS continuous.

Theorem 8. Let $\left(X, \tau_{1}\right)$ and $\left(Y, \tau_{2}\right)$ be two FPFS topological spaces and $f_{u p}: F P F S(X, E) \rightarrow F P F S(Y, K)$ be a FPFS mapping. Then the following are equivalent:

(1) $f_{u p}$ is FPFS continuous,

(2) $f_{u p}^{-1}\left(G_{S}\right)$ is FPFS closed for every FPFS closed set $G_{S}$ over $Y$,

(3) $f_{u p}\left(\overline{F_{A}}\right) \widetilde{\subset} \overline{f_{u p}\left(F_{A}\right)}, \forall F_{A} \in F P F S(X, E)$,

(4) $\overline{f_{u p}^{-1}\left(G_{S}\right)} \widetilde{\subset} f_{u p}^{-1}\left(\overline{G_{S}}\right), \forall G_{S} \in F P F S(Y, K)$,

(5) $f_{u p}^{-1}\left(G_{S}^{\circ}\right) \widetilde{C}\left(f_{u p}^{-1}\left(G_{S}\right)\right)^{\circ}, \forall G_{S} \in F P F S(Y, K)$.

Proof. (1) $\Rightarrow$ (2) It is obvious from Theorem 1 (9).

(2) $\Rightarrow(3)$ Let $F_{A} \in F P F S(X, E)$. Since $F_{A} \widetilde{\subset} f_{u p}^{-1}\left(f_{u p}\left(F_{A}\right)\right), F_{A} \widetilde{\subset} f_{u p}^{-1}\left(\overline{f_{u p}\left(F_{A}\right)}\right) \in \tau_{1}^{\prime}$. Therefore we have $\overline{F_{A}} \widetilde{\subset} f_{u p}^{-1}\left(\overline{f_{u p}\left(F_{A}\right)}\right)$.

By Theorem 1 (4), we get $f_{u p}\left(\overline{F_{A}}\right) \widetilde{\subset} f_{u p}\left(f_{u p}^{-1}\left(\overline{f_{u p}\left(F_{A}\right)}\right) \widetilde{\subset} \overline{f_{u p}\left(F_{A}\right)}\right.$. 
(3) $\Rightarrow(4)$ Let $G_{S} \in F P F S(Y, K)$. If we choose $f_{u p}^{-1}\left(G_{S}\right)$ instead of $F_{A}$ in (3), then $f_{u p}\left(\overline{f_{u p}^{-1}\left(G_{S}\right)}\right) \widetilde{\subset} \overline{f_{u p}\left(f_{u p}^{-1}\left(G_{S}\right)\right)} \widetilde{\subset} \overline{G_{S}}$. Hence by Theorem 1(3), $\overline{f_{u p}^{-1}\left(G_{S}\right)} \widetilde{\subset} f_{u p}^{-1}\left(f_{u p}\left(\overline{\left(f_{u p}^{-1}\left(G_{S}\right)\right.}\right)\right) \widetilde{\subset} f_{u p}^{-1}\left(\overline{G_{S}}\right)$.

(4) $\Leftrightarrow$ (5) These follow from Theorem 1 (9) and Theorem 6.

(5) $\Rightarrow(1)$ Let $G_{S} \in \tau_{2}$. Since $G_{S}$ is a FPFS open set by (5) $f_{u p}^{-1}\left(G_{S}\right)=f_{u p}^{-1}\left(G_{S}^{\circ}\right) \widetilde{\subset} f_{u p}^{-1}\left(G_{S}\right)$. Consequently, $f_{u p}^{-1}\left(G_{S}\right)$ is a FPFS open and so $f_{u p}$ is FPFS continuous.

Theorem 9. Let $f_{u p}:\left(X, \tau_{1}\right) \rightarrow\left(Y, \tau_{2}\right)$ be a FPFS mapping and $\mathscr{B}$ be a base for $\tau_{2}$. Then $f_{u p}$ is FPFS continuous if and only if $f_{u p}^{-1}\left(G_{S}\right) \in \tau_{1}$, for all $G_{S} \in \mathscr{B}$.

Proof. Straightforward.

Definition 21. A family $\mathscr{C}$ of FPFS sets is a cover of a FPFS set $F_{A}$ if $F_{A} \widetilde{\subseteq} \widetilde{\cup}\left\{F_{A_{i}}: F_{A_{i}} \in \mathscr{C}, i \in J\right\}$. It is a FPFS open cover if each member of $\mathscr{C}$ is a FPFS open set. A subcover of $\mathscr{C}$ is a subfamily of $\mathscr{C}$ which is also a cover.

Definition 22. A FPFS topological space $(X, \tau)$ is FPFS-compact if each FPFS open cover of $F_{\widetilde{E}}$ has a finite subcover.,

Example 6. Let $X=\left\{x_{1}, x_{2}, \ldots\right\}, \quad E=\left\{e_{1}, e_{2}, \ldots\right\} \quad$ and $\quad F_{A_{n}}=\left\{\left(\left(e_{1}\right)_{1},\left\{\left(x_{1}\right)_{1}\right\}\right),\left(\left(e_{2}\right)_{\frac{1}{2}},\left\{\left(x_{1}\right)_{1},\left(x_{2}\right)_{\frac{1}{2}}\right\}\right), \ldots\right.$, $\left.\left(\left(e_{n}\right)_{\frac{1}{n}},\left\{\left(x_{1}\right)_{1},\left(x_{2}\right)_{\frac{1}{2}}, \ldots,\left(x_{n}\right)_{\frac{1}{n}}\right\}\right): n=1,2, \ldots\right\}$. Then $\tau=\left\{F_{A_{n}}: n=1,2, \ldots\right\} \cup\left\{F_{\varnothing}, F_{\widetilde{E}}\right\}$ is a FPFS topology on $X$ and $(X, \tau)$ is FPFS-compact.

Definition 23. A family $\mathscr{C}$ of FPFS sets has the finite intersection property if the intersection of the members of each finite subfamily of $\mathscr{C}$ is not empty FPFS set.

Theorem 10. A FPFS topological space is FPFS compact if and only if each family of FPFS closed sets with the finite intersection property has a non empty FPFS intersection.

Proof. If $\mathscr{C}$ is a family of FPFS sets in a FPFS topological space $(X, \tau)$, then $\mathscr{C}$ is a cover of $F_{\widetilde{E}}$ if and only if one of the following conditions holds:

(1) $\widetilde{\cup}\left\{F_{A_{i}}: F_{A_{i}} \in \mathscr{C}, i \in J\right\}=F_{\widetilde{E}}$.

(2) $\left(\widetilde{\cup}\left\{F_{A_{i}}: F_{A_{i}} \in \mathscr{C}, i \in J\right\}\right)^{c}=F_{\widetilde{E}}^{c}=F_{\varnothing}$.

(3) $\widetilde{\cap}\left\{F_{A_{i}}^{c}: F_{A_{i}} \in \mathscr{C}, i \in J\right\}=F_{\varnothing}$.

Hence this shows that FPFS topological space is FPFS compact if and only if each family of FPFS open sets over $X$ such that no finite subfamily covers $F_{\widetilde{E}}$, fails to be a cover, and this is true if and only if each family of FPFS closed sets which has the finite intersection property has a nonempty FPFS intersection.

Theorem 11. Let $\left(X, \tau_{1}\right)$ and $\left(Y, \tau_{2}\right)$ be FPFS topological spaces and $f_{u p}: F P F S(X, E) \rightarrow F P F S(Y, K)$ be a FPFS mapping. If $\left(X, \tau_{1}\right)$ is FPFS compact and $f_{u p}$ is FPFS continuous surjection, then $\left(Y, \tau_{2}\right)$ is FPFS compact.

Proof. Let $\mathscr{C}=\left\{G_{S_{i}}: i \in J\right\}$ be a cover of $G_{\widetilde{K}}$ by FPFS open sets. Then since $f_{u p}$ is FPFS continuous, $\left\{f_{u p}^{-1}\left(G_{S_{i}}: G_{S_{i}} \in \mathscr{C}\right\}\right.$ is a cover of $F_{\widetilde{E}}$ by FPFS open sets. Again since $\left(X, \tau_{1}\right)$ FPFS compact, there exist a finite subset $J_{0}$ of $J$ such that $\left\{f_{u p}^{-1}\left(G_{S_{i}}: i \in J_{0}\right\}\right.$ covers $F_{\widetilde{E}}$. Then we have $f_{u p}\left(\widetilde{\cup}\left\{f_{u p}^{-1}\left(G_{S_{i}}\right): i \in J_{0}\right\}=f_{u p}\left(F_{\widetilde{E}}\right)\right.$ and so $\widetilde{\cup}\left\{G_{S_{i}}: i \in J_{0}\right\}=G_{\widetilde{K}}$. This shows that $\left(Y, \tau_{2}\right)$ is FPFS compact. 


\section{References}

[1] Acar, U., Koyuncu, F. and Tanay, B. Soft sets and soft rings, Comput. Math. Appl. 59, 3458-3463, 2010.

[2] Aktaş, H. and Çağman, N. Soft sets and soft groups, Inform. Sci. 177 (13), 2726-2735, 2007.

[3] Atmaca, S. and Zorlutuna, İ. On fuzzy soft topological spaces, Ann. Fuzzy Math. Inform. 5 (2), 377-386, 2013.

[4] Aygünoğlu, A. and Aygün, H. Introduction to fuzzy soft groups, Comput. Math. Appl. 58, 1279-1286, 2009.

[5] Varol, B. P. and Aygün, H. Fuzzy soft topology, Hacet. J. Math. Stat. 41 (3), 407-419, 2012.

[6] Varol, B. P., Shostak, A. and , H. A new approach to soft topology, Hacet. J. Math. Stat. 41 (5), 731-741, 2012.

[7] Çağman, N., Karataş, S. and Enginoglu, S. Soft topology, Comput. Math. Appl. 62, 351-358, 2011.

[8] Çağman, N., Çıtak, F. and Enginoglu, S. FP set theory and its applications, Ann. Fuzzy Math. Inform. 2 (2), 219-226, 2011.

[9] Çağman, N. and Deli, İ. Products of FP sets and their applications, Hacet. J. Math. Stat. 41 (3), 365-374, 2012.,

[10] N. Cağman and F. Citak and S. Enginoğlu, Fuzzy parameterized fuzzy soft set theory and its applications, Turkish Journal of Fuzzy Systems 1(1) 21-35 (2010).

[11] Çağman, N. and Deli, İ. Means of FP sets and their applications, Hacet. J. Math. Stat. 41 (5), 615-625, 2012.

[12] Çağman, N. and Enginoglu, S. Soft set theory and uni-int decision making, Eur. J. Oper. Res. 207, 848-855, 2010.

[13] Çağman, N. and Enginoglu, S. Soft matrix theory and its decision making, Comput. Math. Appl. 59, 3308-3314, 2010.

[14] Çelik, Y., Ekiz, C. and Yamak, S. A new view on soft rings, Hacet. J. Math. Stat. 40 (2), 273-286, 2011.

[15] Chang, C. L. Fuzzy topological spaces, J. Math. Anal. Appl. 24, 182-190, 1968.

[16] Chen, D., Tsang, E. C. C., Yeung, D. S. and Wang, X. The parametrization reduction of soft sets and its applications, Comput. Math. Appl. 49, 757-763, 2005.

[17] Feng, F., Jun, Y.B. and Zhao, X. Soft semirings, Comput. Math. Appl. 56, 2621-2628, 2008.

[18] Feng, F., Jun, Y.B., Liu, X. and Li, L.F. An adjustable approach to fuzzy soft set based decision making, J. Comput. Appl. Math. 234, 10-20, 2010.

[19] Georgiou, D. N., Megaritis, A.C. and Petropoulos, V.I. On soft topological spaces, Appl. Math. Inf. Sci. (2013), in press.

[20] Inan, E. and Öztürk, M. A. Fuzzy soft rings and fuzzy soft ideals, Neural Comput. and Applic DOI 10.1007/s00521-011-0550-5.

[21] Jun, Y. B. and Park, C.H. Applications of soft sets in ideal theory of BCK/BCI-algebras, Inform. Sci. 178, 2466-2475, 2008.

[22] Kong, Z., Gao, L.Q. and Wang, L.F. Comment on a fuzzy soft set theoretic approach to decision making problems, J. Comput. Appl. Math. 223, 540-542, 2009.

[23] Maji, P. K., Roy, A. R. and Biswas, R. An application of soft sets in desicion making problem, Comput. Math. Appl. 44, 1077-1083, 2002.

[24] Maji, P. K., Biswas, R. and Roy, A. R. Fuzzy soft sets, Journal of Fuzzy Mathematics, 203 (2), 589-602, 2001.

[25] Ming, P. B. and Ming, L. Y. Fuzzy topology I.Neighbourhood structure of a fuzzy point and Moore-Smith convergence, J. Math. Anal. Appl. 76, 571-599, 1980.

[26] Molodtsov, D. Soft set theory-First results, Comput. Math. Appl. 37 (4/5), 19-31, 1999.

[27] Shabir, M. and Naz, M. On soft topological spaces, Comput. Math. Appl. 61, 1786-1799, 2011.

[28] Roy, A.R. and Maji, P.K. A fuzzy soft set theoretic approach to decision making problems, J. Comput. Appl. Math. 203, 412-418, 2007.

[29] Tanay, B. and Kandemir, M. B. Topological structures of fuzzy soft sets, Comput. Math. Appl. 61, 412-418, 2011.

[30] Şimsekler, T. and Yüksel, S. Fuzzy soft topological spaces, Ann. Fuzzy Math. Inform. 5 (1), 87-96, 2013.

[31] Xiao, Z., Gong, K. and Zou, Y. A combined forecasting approach based on fuzzy soft sets, J. Comput. Appl. Math. 228, 326-333, 2009.

[32] Zadeh, L. A. Fuzzy sets, Inform. and Control 8, 338-353, 1965.

[33] Zorlutuna, İ., Akdağ, M., Min, W. K. and Atmaca, S. Remarks on soft topological spaces, Ann. Fuzzy Math. Inform. 3 (2), 171-185, 2011. 